\title{
Estimating the Size of Union of Sets in Streaming Models ${ }^{*}$
}

\author{
Kuldeep S. Meel (r) \\ National University of Singapore
}

\author{
N. V. Vinodchandran $\mathrm{r}$ \\ University of Nebraska, Lincoln
}

\author{
Sourav Chakraborty \\ Indian Statistical Institute, Kolkata
}

\begin{abstract}
In this paper we study the problem of estimating the size of the union of sets $S_{1}, \ldots, S_{M}$ where each set $S_{i} \subseteq \Omega$ (for some discrete universe $\Omega$ ) is implicitly presented and comes in a streaming fashion. We define the notion of Delphic sets to capture class of streaming problems where membership, sampling, and counting calls to the sets are efficient. In particular, we show our notion of Delphic sets capture three well known problems: Klee's measure problem (discrete version), test coverage estimation, and model counting of DNF formulas.
\end{abstract}

The Klee's measure problem corresponds to computation of volume of multi-dimension axis aligned rectangles, i.e., every $d$ dimension axis-aligned rectangle can be defined as $\left[a_{1}, b_{1}\right] \times\left[a_{2}, b_{2}\right] \times$ $\ldots \times\left[a_{d}, b_{d}\right]$. The problem of test coverage estimation focuses on the computation of coverage measure for a given testing array in the context of combinatorial testing, which is a fundamental technique in the context of hardware and software testing. Finally, given a DNF formula $\varphi=T_{1} \vee T_{2} \vee \ldots \vee T_{M}$, the problem of model counting seeks to compute the number of satisfying assignments of $\varphi$.

The primary contribution of our work is a simple and efficient sampling-based algorithm, called APS-Estimator, for estimating the of union of sets in streaming setting. Our algorithm has the space complexity of $O(R \log |\Omega|)$ and update time is $O(R \log R \cdot \log (M / \delta)$. $\log |\Omega|)$ where, $R=O\left(\log (M / \delta) \cdot \varepsilon^{2}\right)$. Consequently, our algorithm provides the first algorithm with linear dependence on $d$ for Klee's measure problem in streaming setting for $d>1$, thereby settling the open problem of Tirthpura and Woodruff (PODS-12).

Furthermore, a straightforward application of our algorithm lends to an efficient algorithm for coverage estimation problem in streaming setting. We then investigate whether the space complexity for coverage estimation can be further improved, and in this context, we present another streaming algorithm that uses near-optimal $O\left(t \log n / \varepsilon^{2}\right)$ space complexity but uses an update algorithm that is in $\mathrm{P}^{\mathrm{NP}}$, thereby showcasing an interesting time vs space trade-off in the streaming setting. Finally, we demonstrate the

*The authors decided to forgo the convention of alphabetical ordering of names in favor of a randomized ordering, denoted by $\mathrm{r}$. The publicly verifiable record of the randomization is available at https://www.aeaweb.org/journals/policies/random-authororder/search. For citation of the work, authors request that the citation guidelines by AEA (available at https://www.aeaweb.org/journals/policies/random-author-order) for random author ordering be followed.

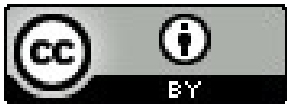

This work is licensed under a Creative Commons Attribution International 4.0 License.

PODS '21, June 20-25, 2021, Virtual Event, China.

(C) 2021 Copyright held by the owner/author(s).

ACM ISBN 978-1-4503-8381-3/21/06.

https://doi.org/10.1145/3452021.3458333 generality of our Delphic sets by obtaining a streaming algorithm for model counting of DNF formulas.

It is worth remarking that we view a key strength of our work is the simplicity of both the algorithm and its theoretical analysis, which makes it amenable to practical implementation and easy adoption.

\section{CCS CONCEPTS}

- Theory of computation $\rightarrow$ Streaming models; Sketching and sampling.

\section{KEYWORDS}

Streaming Algorithms, DNF counting, Klee's Measure Problem

\section{ACM Reference Format:}

Kuldeep S. Meel (r), N. V. Vinodchandran (r), and Sourav Chakraborty. 2021. Estimating the Size of Union of Sets in Streaming Models. In Proceedings of the 40th ACM SIGMOD-SIGACT-SIGAI Symposium on Principles of Database Systems (PODS '21), Fune 20-25, 2021, Virtual Event, China. ACM, New York, NY, USA, 12 pages. https://doi.org/10.1145/3452021.3458333

\section{INTRODUCTION}

Estimating the size of the union of sets is a fundamental problem in Computer Science. The goal, usually, is to design an "efficient" randomized algorithm that can output an $(\varepsilon, \delta)$-approximation of the size of the union of sets. We say that a random variable $Z$ is an $(\varepsilon, \delta)$ approximation of $Y$ if $\operatorname{Pr}[|Z-Y| \leq \varepsilon|Y|] \geq 1-\delta$.

In this paper, we focus on estimating the union of sets in a streaming setting. We consider a family of sets which we call Delphic Sets (see Definition 1.4), for which membership, sampling, and counting queries can be implemented efficiently. To showcase the generality of Delphic sets, we first present three problems arising in diverse domains that can be captured by Delphic sets: Klee's measure problem, test coverage estimation, and model counting for DNF. The three problems are defined as follows:

\section{Klee's Measure Problem}

We define the discrete version of Klee's Measure Problem (KMP) in streaming setting.

Definition 1.1. Ad-dimensional axis aligned rectangle $\boldsymbol{r}$ over an universe $U=[\Delta]^{d}$ is defined as $\left[a_{1}, b_{1}\right] \times\left[a_{2}, b_{2}\right] \times \ldots \times\left[a_{d}, b_{d}\right]$. Given a rectangle $\boldsymbol{r}$, let $\operatorname{Range}(\boldsymbol{r})$ denote set of tuples $\left\{\left(x_{1}, \ldots, x_{d}\right)\right\}$ where $a_{i} \leq x_{i} \leq b_{i}$ and $x_{i}$ is an integer. Note that every $d$-dimensional rectangle can be succinctly represented by the tuple $\left(a_{1}, b_{1}, \cdots a_{d}, b_{d}\right)$. Given a stream $\mathcal{R}$ of size $M$ such that $\mathcal{R}=\left\langle\boldsymbol{r}_{1}, \boldsymbol{r}_{2}, \cdots \boldsymbol{r}_{M}\right\rangle$, where each item $\boldsymbol{r}_{i}$ is a d-dimensional rectangle, we are interested in computing $a$ $(\varepsilon, \delta)$-approximation of the volume of $\mathcal{R}$, which is defined as follows:

$$
\operatorname{Volume}(\mathcal{R})=\left|\cup_{1 \leq i \leq M} \operatorname{Range}\left(\boldsymbol{r}_{i}\right)\right|
$$


Practical Motivation. Klee's Measure Problem is a well-investigated problem in computational geometry. Klee in 1977 introduced the one-dimensional version of the problem over reals: given $n$ intervals in $\mathbf{R}$, compute the size of their union [32]. Klee presented $O(n \log n)$ time algorithm, which was later proved to be optimal by Fredmen and Weide [24]. Since its introduction, this problem has been studied extensively in computational geometry with the goal of designing efficient algorithms in the traditional RAM model. As certain data objects in databases can be represented by axis parallel multi-dimension rectangles, the KMP problem is significant in data bases [10, 34, 44, 53, 59]. In addition to computer science areas, algorithms for KMP have recently found applications in a varied range of practical areas including in environmental chemistry [20] and lunar archaeology [6].

The discrete version of KMP that we consider in this paper is studied in the streaming community as range efficient $\mathbb{F}_{0}$ computation $[45,56]$. Other than its natural appeal, this problem is interesting because several significant problems, including maxdominance norm [21], counting triangles in graphs [3], and distinct summation problem [19] can be reduced to computing $\mathbb{F}_{0}$ over multi-dimensional discrete ranges.

\section{Test Coverage Estimation Problem}

Definition 1.2. For an $n$-bit binary string $\boldsymbol{a}=a_{1} a_{2} \cdots a_{n} \in\{0,1\}^{n}$, the $t$-coverage, denoted by $\operatorname{Cov}_{t}(\boldsymbol{a})$, is defined as

$$
\begin{aligned}
\operatorname{Cov}_{t}(\boldsymbol{a}) & =\left\{(T, \boldsymbol{y})|T \subset[n],| T \mid=t, \boldsymbol{y} \in\{0,1\}^{t}\right. \\
& \text { and the restriction of } \left.a_{i} \text { to indices in } T \text { gives } \boldsymbol{y}\right\}
\end{aligned}
$$

The input is a stream $\mathcal{A}$ of size $M$ such that $\mathcal{A}=\left\langle\boldsymbol{a}_{1}, \ldots, \boldsymbol{a}_{M}\right\rangle$ where $\boldsymbol{a}_{i} \in\{0,1\}^{n}$, the $t$-coverage of $\mathcal{A}$ is

$$
\operatorname{Cov}_{t}(\mathcal{A})=\cup_{1 \leq i \leq M} \operatorname{Cov}_{t}\left(\boldsymbol{a}_{i}\right) .
$$

The coverage estimation problem is: Given a stream $\mathcal{A}=\mathbf{a}_{1}, \cdots, \mathbf{a}_{M}$, compute an $(\varepsilon, \delta)$-approximation of $\left|\operatorname{Cov}_{t}(\mathcal{A})\right|$ for any given $t$.

Practical Motivation. Over the past half-century, the widespread adoption of software in diverse areas has necessitated the design of highly configurable systems wherein the end-user can choose a configuration of interest by setting the desired values to the configuration parameters. The space of configurations is often astronomical, and perhaps can be best illustrated by the observation that the possible number of configuration options of an embedded Linux for microcontrollers is $7.7 \times 10^{417}$ possible configurations [47]. Since it is not feasible to examine the behavior of a System Under Test (SUT) for all possible configurations, the combinatorial testing has emerged as a dominant paradigm [33]. A configuration is specified by assigning values to the configuration parameters ${ }^{1}$. Motivated by the observation that often the errors in systems result due to the interaction of a small number of parameters, the paradigm of combinatorial testing focuses on covering as many $t$-combinations of parameters. Note that when each of the parameters takes binary values, the total number of possible $t$-combinations of parameters over a test of size $n$ is $\left(\begin{array}{c}n \\ t\end{array}\right) 2^{t}$.

\footnotetext{
${ }^{1}$ While techniques developed in our work extends to scenarios wherein each of the parameters take values in finite domains, for simplicity of exposition, we will focus on the case where each of the parameters take a binary value.
}

Given the critical importance of software testing, there has been a long line of work in the design of test suite generators $[9,18$, $35,37,42,54,55]$. The earliest works focused on the design of the smallest size of test suites such that all the $\left(\begin{array}{c}n \\ t\end{array}\right) 2^{t}$ combinations are covered. The optimal constructions still generate test suites of size $O\left(t 2^{t} \log n\right)$, which is intractable for large enough $t$. Consequently, one is often interested in achieving as high a coverage as possible within a given budget. Moreover, such generators are heuristicbased and fail to provide any rigorous guarantees on the quality of their generated test suites. In this context, it is critical to rigorously estimate the coverage given a set of tests. Note that in the context of binary parameters, a test can be specified as a binary string $\mathbf{a}=$ $a_{1} a_{2} \ldots a_{n} \in\{0,1\}^{n}$. For practical systems, $n$ is often very large, and therefore, it is not practical to store all the tests. In addition, it is useful to have estimation methods that deal with a growing test suit. In particular, from a practical perspective, one envisions a coverage estimator to be a monitor with as small resource overhead as possible. These issues lend themselves to modeling coverage estimation problem in a data streaming framework.

\section{Model Counting for DNF}

Definition 1.3. Consider a set $X$ of $n$ Boolean variable. A literal is a variable or its negation. A formula $\varphi$ over $X$ is in DNF if it is represented as disjunction over conjunction of literals. Each such conjunction is called a term, therefore, $\varphi$ over $M$ terms is represented as $T_{1} \vee T_{2} \vee \ldots \vee T_{M}$. Let $\operatorname{Sol}(\varphi)$ represent the set of satisfying assignments of $\varphi$. For a DNF formula $\varphi=T_{1} \vee T_{2} \vee \ldots \vee T_{M}$, given the terms as a stream $\left\langle T_{1}, \ldots, T_{M}\right\rangle$, we are interested in computation of an $(\varepsilon, \delta)$-approximation of $|\operatorname{Sol}(\varphi)|$.

The problem of model counting for DNF, also referred to as DNF counting, is also often denoted by \#DNF.

Practical Motivation. \#DNF is a fundamental problem in computer science with a wide variety of applications. Dalvi and Suici [23] showed that queries in probabilistic databases reduce to \#DNF. Another important application of \#DNF arises from the domain of network unreliability: given a graph $G=(V, E)$, wherein each edge $e_{i}$ fails with probability $p_{i}$, we are interested in the computation of the probability that $s$ and $t$ are disconnected. Karger's seminal work [29] reduces the computation of network unreliability to \#DNF wherein each term represents a min-cut. The past few years have witnessed a surge in interest for designing efficient FPRAS techniques for \#DNF [39, 40].

\subsection{Our Results}

We first define the notion of Delphic sets (Definition 1.4). The corresponding problem in the streaming model (Problem 1.5) helps to capture all the above three problems, and potentially many other union-of-sets problems, under a general framework. Then (in Theorem 1.6) we present an efficient algorithm for estimating the size of the union of a stream of Delphic sets. We measure the efficiency of our algorithm both in terms of worst case space complexity and worst case per-item update time. Finally we show how our algorithm for Delphic sets gives new efficient algorithms for all the above three problems. The algorithm for KMP solves an open problem from the literature. 


\section{Delphic Sets as a Unifying Model}

Let $\Omega$ be a discrete universe. We define the notion of Delphic family as follows:

Definition 1.4. A set $S \subseteq \Omega$ belongs to Delphic family if the following queries can be done in $O(\log |\Omega|)$ time.

(1) Know the size of the set $S$,

(2) Draw a uniform random sample from $S$, and

(3) Given any $x$ check if $x \in S$.

Next, we define the following streaming problem;

Problem 1.5. Given a stream $\mathcal{S}=\left\langle S_{1}, S_{2}, \ldots, S_{M}\right\rangle$ wherein each $S_{i}$ belongs to Delphic family, and $0<\varepsilon, \delta<1$ output an $(\varepsilon, \delta)$ approximation of $\left|\bigcup_{i=1}^{M} S_{i}\right|$.

Taking a departure from standard sketching based techniques, we develop an adaptive sampling-based algorithm for Delphic unionof-sets problem, as stated in the following theorem.

THEOREM 1.6. There is a streaming algorithm APS-Estimator that given any reals numbers $\varepsilon, \delta<1$, and a stream $\mathcal{S}=\left\langle S_{1}, S_{2} \cdots, S_{M}\right\rangle$ wherein each $S_{i} \subseteq \Omega$ belongs to Delphic family, computes an $(\varepsilon, \delta)$ approximation of $\left|\bigcup_{i=1}^{M} S_{i}\right|$. The algorithm has worst case space complexity $O(R \log |\Omega|)$ and update time is $O(R \log R \cdot \log (M / \delta) \cdot \log |\Omega|)$ where, $R=O\left(\log (M / \delta) \cdot \varepsilon^{-2}\right)$.

Remark 1.7. Tirthapura and Woodruff [56] (PODS-12) employed the notation $O^{*}(f)$ as a place holder for $O\left(f \cdot\left(\varepsilon^{-1} \cdot \log \left(\frac{|\Omega| M}{\delta}\right)\right){ }^{O(1)}\right)$. Therefore, we can state the space and update time complexity of APS-Estimator as $O^{*}(1)$ in Tirthapura and Woodruff's notations.

\section{Klee's Measure Problem}

We first observe that Klee's measure problem can be formulated as Delphic coverage by constructing set $S_{i}=\operatorname{Range}\left(r_{i}\right)$ corresponding to each $\mathbf{r}_{i}$ and observing that each such $S_{i}$ belongs to the Delphic family. Therefore, the following Corollary follows from Theorem 1.6

Corollary 1.8. There is a streaming algorithm that given any reals numbers $\varepsilon, \delta<1$, and a stream $\mathcal{R}=\left\langle\boldsymbol{r}_{1}, \boldsymbol{r}_{2}, \cdots \boldsymbol{r}_{M}\right\rangle$ where each $\boldsymbol{r}_{i}$ is a d-dimensional rectangle over $\Omega=\left[\Delta^{d}\right]$, computes an $(\varepsilon, \delta)$ approximation of $\operatorname{Volume}(\mathcal{R})$. The algorithm has worst case space $O\left(d(\log \Delta) \cdot \log (M / \delta) \cdot \varepsilon^{-2}\right)$ and update time complexity $O\left(d(\log \Delta) \cdot(\log (M / \delta))^{2} \log \log (M / \delta) \cdot \varepsilon^{-2} \log \varepsilon^{-1}\right)$.

Remark 1.9. Corollary 1.8 provides the first efficient algorithm with linear dependence on d for Klee's measure problem in a streaming setting, thereby resolving the open problem from Tirthapura and Woodruff [56]. In this context, it is worth remarking that Tirthapura and Woodruff claimed an algorithm for KMP with space and update time complexity $O\left(d(\log \Delta) \cdot(\log M) \cdot \varepsilon^{-2} \cdot \log \left(\frac{1}{\delta}\right)\right)$. However, they have retracted their claim [58]. Their method only yields update time complexity of poly $\left((\log \Delta)^{d}, \varepsilon^{-1}, \log \frac{1}{\delta}\right)$.

Multi-Dimensional Arithmetic Progression. We then focus on generalization of Klee's measure problem by generalizing the notion of range $\left[a_{i}, b_{i}\right]$ to arithmetic progressions, which was studied previously by Pavan and Tirthapura [45] for $d=1$. Let $[a, b, c]$ represent the arithmetic progression with common difference $c$ in the range $[a, b]$, i.e., $a, a+c, a+2 c, a+j c$, where $j$ is the largest integer such that $a+j c \leq b$. Consider a stream $\mathcal{R}=\left[r_{1}, r_{2}, \ldots r_{m}\right]$ wherein each $r_{i}=\left[a_{1}, b_{1}, c_{1}\right] \times \cdots \times\left[a_{d}, b_{d}, c_{d}\right]$. each $r_{i}=\left[a_{1}, b_{1}, c_{1}\right] \times$ $\cdots \times\left[a_{d}, b_{d}, c_{d}\right]$. We generalize Range $(r)$ to denote the set of tuple $\left\{\left(x_{1}, \ldots x_{d}\right)\right\}$ where $a_{i} \leq x_{i} \leq b_{i}$ and $x_{i}=a_{i}+k \cdot c_{i}$ for some positive integer k. Similarly, Volume $(\mathcal{R})=\left|\bigcup_{i=1}^{m} \operatorname{Range}\left(r_{i}\right)\right|$. By observing that Range $\left(r_{i}\right)$ for $d$-dimensional arithmetic progress belongs to Delphic family, we obtain the following streaming algorithm.

Corollary 1.10. There is a streaming algorithm that given any positive reals numbers $\varepsilon, \delta<1$, and a stream $\mathcal{R}=\left\langle\boldsymbol{r}_{1}, \boldsymbol{r}_{2}, \cdots \boldsymbol{r}_{M}\right\rangle$ consisting of d-dimensional arithmetic progressions, computes an $(\varepsilon, \delta)$ approximation of $\operatorname{Volume}(\mathcal{R})$. The algorithm has worst case space complexity $O\left(d(\log \Delta) \cdot \log (M / \delta) \cdot \varepsilon^{-2}\right)$ and worst case update time complexity $O\left(d(\log \Delta) \cdot(\log (M / \delta))^{2} \log \log (M / \delta) \cdot \varepsilon^{-2} \log \varepsilon^{-1}\right)$.

Observe that Klee's measure problem is a special case of multidimensional arithmetic progress wherein $c_{i}=1$. It is worth remarking that in comparison to prior work that focused on the special case of arithmetic progress for $\mathrm{d}=1$, the algorithm for multidimensional ranges (i.e., Klee's measure problem) can be simply lifted to multi-dimensional arithmetic progression, and the space and time complexity does not change.

\section{Test Coverage Estimation}

Next, we observe that Test Coverage Estimation problem can be also formulated as Delphic coverage by constructing $S_{i}=\operatorname{Cov}\left(\mathbf{a}_{i}\right)$ for each $\mathbf{a}_{i}$, and again observing that each such $S_{i}$ belongs to Delphic family.

Corollary 1.11. There is a streaming algorithm APS-Estimator that given any reals numbers $\varepsilon, \delta<1$, and a stream $\mathcal{A}=\left\langle\boldsymbol{a}_{1}, \cdots, \boldsymbol{a}_{M}\right\rangle$, where $\boldsymbol{a}_{i} \in\{0,1\}^{n}$, computes an $(\varepsilon, \delta)$-approximation of $\left|\operatorname{Cov}_{t}(\mathcal{A})\right|$. The algorithm has space complexity $O\left(t(\log n) \cdot \log (M / \delta) \cdot \varepsilon^{-2}\right)$ and worst case update time complexity

$$
O\left(t(\log n) \cdot(\log (M / \delta))^{2} \log \log (M / \delta) \cdot \varepsilon^{-2} \log \varepsilon^{-1}\right) .
$$

We also investigate whether the space complexity can be further improved in the context of test coverage estimation. We present a hashing-based algorithm that trades off improvement in space complexity with an increase in the overhead for time complexity via relying on the usage of NP oracles.

THeOREM 1.12. There is a streaming algorithm HashingEstimator with an NP set as an oracle that given a stream $\mathcal{A}=\left\langle\boldsymbol{a}_{1}, \cdots, \boldsymbol{a}_{M}\right\rangle$, and real numbers $0<\varepsilon, \delta<1$, where each $\boldsymbol{a}_{i} \in\{0,1\}^{n}$ computes an $(\varepsilon, \delta)$-approximation of $\left|\operatorname{Cov}_{t}(\mathcal{A})\right|$. The algorithm takes $O(t \log n$. $\left.\varepsilon^{-2} \cdot \log \frac{1}{\delta}\right)$ space and $\operatorname{poly}(n, t, 1 / \varepsilon, \log 1 / \delta)$ update time. Thus, if $\mathrm{P}=\mathrm{NP}$, algorithm will run in time $\operatorname{poly}(n, t, 1 / \varepsilon, \log 1 / \delta)$.

The space complexity of the above algorithm, in general, is tight up to a $O(\log n)$ factor as for $t=n$. The problem reduces the problem of $\mathbb{F}_{0}$ computation in the traditional insertion-only data streams for which a lower bound of $\Omega\left(n+\varepsilon^{-2}\right)$ is known [28] (notice that in our case items are from $\left.\{0,1\}^{n}\right)$. Therefore, the problem of designing algorithms that achieve tight bounds from both space and time complexity perspective remains open. 


\section{DNF Counting}

We again observe that DNF counting can be formulated as Delphic coverage by constructing set $S_{i}=\operatorname{Sol}\left(T_{i}\right)$ corresponding to each term $T_{i}$, and observing that each $S_{i}$ belongs to Delphic family. Therefore, the following corollary follows from Theorem 1.6.

Corollary 1.13. There is a streaming algorithm that given any positive reals numbers $\varepsilon, \delta<1$, and a stream $\left\langle T_{1}, T_{2}, \ldots T_{M}\right\rangle$ of terms over $n$ variables, computes an $(\varepsilon, \delta)$-approximation of $\mid \operatorname{Sol}(\varphi \mid)$ wherein $\varphi=T_{1} \vee T_{2} \vee \ldots \vee T_{M}$. The algorithm takes $O\left(n \cdot \log (M / \delta) \cdot \varepsilon^{-2}\right)$ space and $O\left(n \cdot(\log (M / \delta))^{2} \log \log (M / \delta) \cdot \varepsilon^{-2} \log \varepsilon^{-1}\right)$ update time.

\subsection{Techniques}

Our main algorithm, APS-Estimator, is a simple and elegant samplingbased algorithm. But to help in the presentation and analysis, we will present it in two stages.

First, we present algorithm FPS-Estimator (FPS stands for fixed probability sampling-based). The main idea is to sample each item from $\cup_{i} S_{i}$ independently with probability $p$, a number that is proportional to the reciprocal a parameter $L$. The algorithm has space and update time complexity

$$
O\left(\left|\cup_{i} S_{i}\right| \cdot \frac{\log (1 / \delta)+\log M}{L \varepsilon^{2}} \cdot(\log |\Omega|)\right),
$$

and guarantee that the output is an $(\varepsilon, \delta)$-approximation of $\left|\cup_{i} S_{i}\right|$ if $\left|\cup_{i} S_{i}\right|$ is at least $L$. The estimator's elegance lies in the simple procedure that helps to ensure that each item from $\cup_{i} S_{i}$ is independently sampled with a fixed probability as the stream arrives.

In the second (and our main) algorithm APS-Estimator (APS stands for adaptive probability sampling-based), the basic concept is similar to that of FPS-Estimator, but the probability of sampling is adaptively updated during the run of the algorithm. The most crucial observation is that if we could have obtained a good lower bound on the size of $\cup_{i} S_{i}$, then by using that number as $L$ in FPS-Estimator we would have obtained a $(\varepsilon, \delta)$-approximation of $\left|\cup_{i} S_{i}\right|$. Now since the space and update time complexity of FPS-Estimator is inversely proportional to $L$, a better guarantee on the lower bound of $\left|\cup_{i} S_{i}\right|$ would give an improved space and update time complexity.

In APS-Estimator as the stream arrives the lower bound for $\mid \cup_{i}$ $S_{i} \mid$ is continuously estimated. And accordingly, the probability for sampling goes down as the algorithm proceeds. Using a simple re-sampling procedure APS-Estimator ensure that each item from $\cup_{i} S_{i}$ is independently sampled with the same probability $p$, even as the value of $p$ may go down as the stream arrives. This also ensures that the $\left|\cup_{i} S_{i}\right| / L$ factor is shaved off from the sample complexity (and in turn, the space and update time complexity) of FPS-Estimator.

Note that for the sake of presenting a unified framework, we derive time complexity solely based on the three properties of Delphic sets, which allows us only black-box access to a random sample. The time complexity analysis requires generation of samples without repetition, which can be be performed more efficiently in specific contexts of $\operatorname{Cov}\left(\mathbf{a}_{i}\right)$, Range $\left(r_{i}\right)$, $\operatorname{Sol}\left(T_{i}\right)$. We leave a refined analysis of the time complexity for specific problems to the full version.
We view a key strength of our work is the simplicity of both the algorithm, APS-Estimator, and its theoretical analysis. Our algorithm's simplicity makes it amenable to practical implementation, while our analysis's simplicity lends itself to easy verification and adoption.

Organization. The rest of the paper is organized as follows: We discuss related work in Section 2. We then discuss notations and preliminaries in Section 3. The paper's primary technical contribution is presented in Section 4, which consists of four parts. Section 4.1 provides the proof of the main technical theorem 1.6. We then demonstrate Theorem 1.6 can be applied in different contexts, Klee's Measure Problem (Section 4.2), coverage estimation (Section 4.3), and DNF counting (Section 4.4) to obtain efficient streaming algorithms. We finally conclude in Section 5.

\section{RELATED WORK}

Starting with the seminar work of Alon, Matias, and Szegedy [2], the streaming model of computation has emerged as an important area of research in theoretical computer science. This model is well suited to investigate algorithmic problems that arise from real life situations dealing with large data. A central focus of investigation has been on estimating the frequency moments $\mathbb{F}_{k}$ of a stream of data items. In particular, considerable work has been done in designing algorithms for estimating the the $0^{\text {th }}$ frequency moment $\left(\mathbb{F}_{0}\right)$, the number of distinct elements in the stream, culminating in the development of an algorithm with optimal space complexity $O\left(\log |\Omega|+\frac{1}{\varepsilon^{2}}\right)$ and $O(1)$ update time [28], where $\Omega$ is the universe.

In the case when items in the data stream succinctly represent a set of elements of the universe, $\mathbb{F}_{0}$ estimation becomes estimation of size of the union of sets. The union-of-sets problem has been studied in approximate counting literature. The line of work that is closest to ours is the work on DNF counting problem initiated by Karp and Luby [30]. Since the work of Karp and Luby, substantial research has gone into understanding various aspects of DNF counting problem including designing hashing-based algorithms [13, 22, 31, 39-41]. We note that Karp-Luby algorithm can be adapted to counting union of sets in the streaming setting to get an algorithm with space and time complexity $O\left(\frac{M \log |\Omega|}{\epsilon^{2}} \log M \log n\right)$. In comparison, we achieve only a logarithmic dependence on $M$.

As mentioned in the introduction, Klee's Measure Problem (KMP) is a fundamental problem that is well investigated in computational geometry with the focus of designing efficient algorithms in the traditional RAM model. Klee introduced the one-dimensional version of the problem over reals and presented $O(n \log n)$ time algorithm where $n$ is the number of line segments [32]. Fredmen and Weide showed that this is optimal in time (under certain model) [24]. Since then substantial work has gone into extending the algorithms to multidimensional case $[5,8,14,16,17,26,43]$ and also with space complexity considerations $[16,57]$.

Discrete version of KMP over streaming model has been considered before. However the success has been limited [3, 45, 52, 56]. Pavan and Thirthapura considered the problem for one dimensional ranges over the discrete domain $\{1, \ldots, n\}$ and gave an algorithm with $O\left(\varepsilon^{-2} \log n \log 1 / \delta\right)$ space and $O(\log n / \varepsilon \log 1 / \delta)$ update time [45]. Sharma, Busch, Vaidyanathan, Rai, and Trahan 
considered the two-dimensional version but only gave a $O(\sqrt{\log U})$ approximation for the general case where $U$ is the total number of discrete points in the space [48]. Thirthapura and Woodruff [56] considered the general $d$ dimensional problem. They presented an algorithm, based of range efficient implementations of count sketch algorithm [15] and recursive sketches [7, 27], which is efficient in space complexity. However the update time of the algorithm has exponential dependency on the dimension $d$ (see Remark 1.9). In a concurrent work, Pavan (r) Vinodchandran (r) Bhattacharyya (r) $\mathrm{Meel}^{2}$ [46] also proposed another hashing-based technique with exponential dependence on the dimension $d$.

\section{NOTATIONS AND PRELIMINARIES}

We will denote by $[n]$ the set $\{1,2, \ldots, n\}$ and by $\left(\begin{array}{c}{[n]} \\ t\end{array}\right)$ the set of all subsets of $[n]$ of size $t$. For any $n \in \mathbb{N}$ and any $p \in[0,1]$ we will also use $B(n, p)$ to denote the binomial distribution over the set of natural numbers $\{0, \ldots, n\}$ where probability of a number $0 \leq m \leq n$ is $\left(\begin{array}{c}n \\ m\end{array}\right) p^{m}(1-p)^{n-m}$.

At any point the input item is a length $n$ string. However, as done in the case of traditional space bounded computations, for counting space, we will not include the space required to represent the input item. We will consider that input is available on a readonly input tape and do not contribute to the space used by the algorithm. In this paper we consider unit-cost model and assume all basic operations including arithmetic operations on words can be performed in unit time.

\subsection{Concentration Inequalities}

Our technical analysis employs the standard concentration inequalities: Chernoff bound, Chebychev's bound, and Paley-Zygmund Inequality.

Theorem 3.1 (Chernoff Bound). Suppose $v_{1}, \ldots, v_{n}$ are independent random variables taking values in $\{0,1\}$. Let $V=\sum_{i=1}^{n} v_{i}$ and $\mu=\mathbb{E}[V]$ then

$$
\operatorname{Pr}(|V-\mu| \geq \delta \mu) \leq 2 e^{-\frac{\delta^{2} \mu}{3}}
$$

Theorem 3.2 (Chebychev's Inequality). Let $Z$ be a random variable with expected value $\mu$ and non-zero variance $\sigma^{2}$. Then for any real number $k>0$,

$$
\operatorname{Pr}(|Z-\mu| \geq k \sigma) \leq \frac{1}{k^{2}} .
$$

Theorem 3.3 (PALEyÂĂŞZygmund Inequality). If $Z \geq 0$ is $a$ random variable with finite variance, and if $0 \leq \theta \leq 1$, then

$$
\operatorname{Pr}(Z>\theta \mathbb{E}[Z]) \geq(1-\theta)^{2} \frac{\mathbb{E}[Z]^{2}}{\mathbb{E}\left[Z^{2}\right]}
$$

\subsection{Coupon Collector Problem}

For the proof of Theorem 1.6 we will need the following theorem, popularly known as the Coupon Collector Problem.

THeOREM 3.4. Given access to uniform random samples from a set $T$ and $a$ number $r \leq|T|$, let $Z_{r}$ be a random variable that stand for

\footnotetext{
${ }^{2}$ () refers to the randomized author ordering.
}

the number of independent uniform random samples from $T$ needed before we get $r$ distinct samples from $T$. Then

$$
\operatorname{Pr}\left[Z_{r}>\beta r \log r\right] \leq r^{-\beta+1} .
$$

\subsection{Pairwise Independent Hash functions}

Let $n, m \in \mathbb{N}$ and $\mathcal{H}(n, m) \triangleq\left\{h:\{0,1\}^{n} \rightarrow\{0,1\}^{m}\right\}$ be a family of hash functions mapping $\{0,1\}^{n}$ to $\{0,1\}^{m}$. We use $h \stackrel{R}{\leftarrow} \mathcal{H}(n, m)$ to denote the probability space obtained by choosing a function $h$ uniformly at random from $\mathcal{H}(n, m)$.

Definition 3.5. A family of hash functions $\mathcal{H}(n, m)$ is 2-wise independent if $\forall \alpha_{1}, \alpha_{2} \in\{0,1\}^{m}$, distinct $x_{1}, x_{2}, \in\{0,1\}^{n}, h \stackrel{R}{\longleftarrow}$ $\mathcal{H}(n, m)$,

$$
\operatorname{Pr}\left[\left(h\left(x_{1}\right)=\alpha_{1}\right) \wedge\left(h\left(x_{2}\right)=\alpha_{2}\right)\right]=\frac{1}{2^{2 m}}
$$

Explicit families. In this work, one hash family of particular interest is $\mathrm{H}_{\text {Teop }}(n, m)$, which is known to be 2-wise independent [11]. The family is defined as follows: $\mathrm{H}_{\text {Teop }}(n, m) \triangleq\left\{h:\{0,1\}^{n} \rightarrow\right.$ $\left.\{0,1\}^{m}\right\}$ is the family of functions of the form $h(x)=A x+b$ with $A \in \mathbb{F}_{2}^{m \times n}$ and $b \in \mathbb{F}_{2}^{m \times 1}$ where $A$ is a uniformly randomly chosen Toeplitz matrix of size $m \times n$ while $b$ is uniformly randomly matrix of size $m \times 1$. it is worth noticing that $\mathrm{H}_{\text {Teop }}$ can be represented with $\Theta(n)$-bits.

For every $m \in\{1, \ldots n\}$, the $m^{t h}$ prefix-slice of $h$, denoted $h_{m}$, is a map from $\{0,1\}^{n}$ to $\{0,1\}^{m}$, where $h_{m}(y)$ is the first $m$ bits of $h(y)$. Observe that when $h(x)=A x+b, h_{m}(x)=A_{m} x+b_{m}$, where $A_{m}$ denotes the submatrix formed by the first $m$ rows of $A$ and $b_{m}$ is the first $m$ entries of the vector $b$.

\section{THE ALGORITHMS}

\subsection{Coverage of Delphic Sets}

We restate the theorem that we prove in this section.

THEOREM 1.6. There is a streaming algorithm APS-Estimator that given any reals numbers $\varepsilon, \delta<1$, and a stream $\mathcal{S}=\left\langle S_{1}, S_{2} \cdots, S_{M}\right\rangle$ wherein each $S_{i} \subseteq \Omega$ belongs to Delphic family, computes an $(\varepsilon, \delta)$ approximation of $\left|\cup_{i=1}^{M} S_{i}\right|$. The algorithm has worst case space complexity $O(R \log |\Omega|)$ and update time is $O(R \log R \cdot \log (M / \delta) \cdot \log |\Omega|)$ where, $R=O\left(\log (M / \delta) \cdot \varepsilon^{-2}\right)$.

As discussed in Section 1.2 we will prove Theorem 1.6 in two stages. In the first stage we will present a simple sample based algorithm (we call it FPS-Estimator - FPS stands for fixed probability sampling-based). In the next stage we improve upon the FPS-Estimator algorithm and present the APS-Estimator algorithm that is would prove Theorem 1.6.

\subsubsection{Algorithm FPS-Estimator.}

We prove the following theorem.

THEOREM 4.1. There is a streaming algorithm FPS-Estimator that given any reals numbers $\varepsilon, \delta<1$, and a stream $\mathcal{S}=\left\langle S_{1}, S_{2} \cdots, S_{M}\right\rangle$ wherein each $S_{i} \subseteq \Omega$ belongs to Delphic family, computes an $(\varepsilon, \delta)$ approximation of $\left|\bigcup_{i=1}^{M} S_{i}\right|$ assuming $\left|\bigcup_{i=1}^{M} S_{i}\right|$ is at least $L$ (a parameter). With probability at least $(1-\delta / 2)$ the algorithm has space complexity $O(R \cdot \log |\Omega|)$ and update time complexity $O(R \log R$. 
$\log (M / \delta) \cdot \log |\Omega|)$ where

$$
R=O\left(\left|\cup_{i} S_{i}\right| \cdot \frac{\log (1 / \delta)+\log M}{L \varepsilon^{2}}\right) .
$$

We present the algorithm below and establish its correctness.

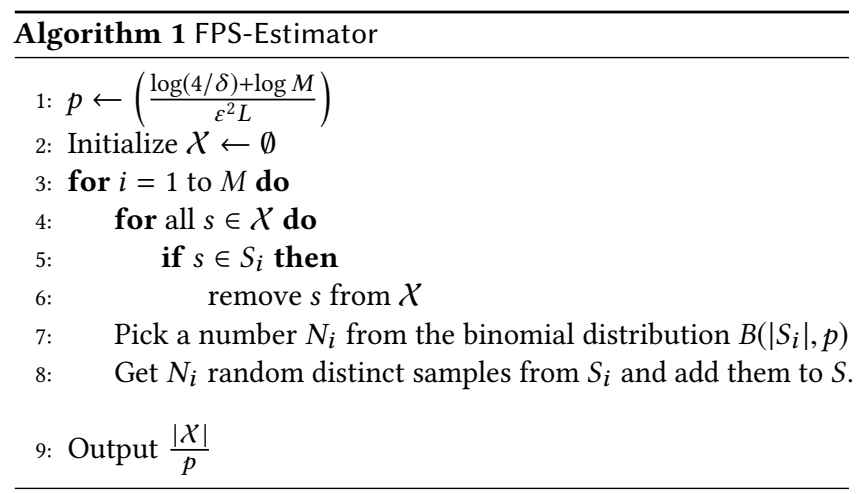

Claim 4.2. Let $R$ be a set of $N$ elements and each element of $R$ is selected independently with probability p. Let $P$ be the random variable that counts the number of selected items. Then

$$
\operatorname{Pr}[(1-\varepsilon) p N \leq P \leq(1+\varepsilon) p N] \geq 1-2 e^{-\varepsilon^{2} p N}
$$

Proof. Follows from simple application of the Chernoff Bound.

Claim 4.3. In Algorithm 1 every element of $\bigcup_{i=1}^{M} S_{i}$ is selected in $\mathcal{X}$ independently with probability $p$.

Proof. Before we start the prove of the Claim, it is important to observe that in line 7-8 all we are doing is drawing each element of $S_{i}$ with probability $p$. This is because drawing each element of $S_{i}$ with probability $p$ can be simulated by first identifying how many distinct elements will be picked from $S_{i}$ (and this is done by drawing a number $N_{i}$ from the binomial distribution $\left.B\left(\left|S_{i}\right|, p\right)\right)$ and then drawing that many distinct elements from $S_{i}$ (by drawing uniform samples from $S_{i}$ until we have $N_{i}$ distinct samples from $S_{i}$.

Now let us prove the claim. For any $j$, define $\widetilde{S_{j}}$ as

$$
\widetilde{S_{j}}=S_{j} \backslash \cup_{j+1 \leq i \leq M} S_{i} .
$$

Consider any element $x \in \bigcup_{i=1}^{M} S_{i}$. We will argue that at the end of the algorithm $x$ is in $\mathcal{X}$ with probability $p$.

Notice that $x \in \widetilde{S_{j}}$ for a unique $j$. Without loss of generality let us assume that $x \in \widetilde{S_{j}}$. Consider the outermost for loop in Algorithm 1 when $S_{j}$ is considered. Note that even if $x$ may be in the set $X$ in the for loop from line $4-6$, it would be removed from $\mathcal{X}$. Now, in the for loop in line 7-8, $x$ would be included in the set $\mathcal{X}$ with probability $p$, and this selection is independent of any other event. Finally note that once $x$ is selected or not selected its status is never changed with respect to inclusion in $X$ because $x$ is not in $\cup_{j+1 \leq i \leq M} S_{i}$.

Lemma 4.4. If $\left|\cup_{i} S_{i}\right|$ is at least $L$, then with probability $\geq(1-$ $\delta / 2)$ the output of FPS-Estimator is between $(1-\varepsilon)\left|\cup_{i} S_{i}\right|$ and $(1+$ $\varepsilon)\left|\bigcup_{i} S_{i}\right|$ and the maximum size of $\mathcal{X}$ during the whole run of the algorithm is $O\left((1+\varepsilon) \cdot\left(\left|\bigcup_{i} S_{i}\right|\right) \cdot \frac{\log (1 / \delta)+\log M}{L \varepsilon^{2}}\right)$.
Proof. From Claim 4.2 and Claim 4.3, the probability that $|X|$ is between $(1-\varepsilon) p\left|\bigcup_{i} S_{i}\right|$ and $(1+\varepsilon) p\left|\bigcup_{i} S_{i}\right|$ is at least

$$
\left(1-2 e^{-\varepsilon^{2} p\left|\cup_{i} S_{i}\right|}\right) \text {. }
$$

Since $\left|\cup_{i} S_{i}\right|$ is at least $L$ so

$$
e^{-\varepsilon^{2} p\left|\bigcup_{i} S_{i}\right|} \leq e^{-(\log (4 / \delta)+\log M)}=\frac{\delta}{4 M} .
$$

So at the end with probability at least $\left(1-\frac{\delta}{2 M}\right)|X|$ is between $(1-\varepsilon) p\left|\bigcup_{i} S_{i}\right|$ and $(1+\varepsilon) p\left|\bigcup_{i} S_{i}\right|$.

This proves that FPS-Estimator outputs an $(\varepsilon, \delta)$-approximation of $\left|\bigcup_{i} S_{i}\right|$.

But note that, the size of $\mathcal{X}$ can increase and decrease during the run of the algorithm. So to prove that the size of $X$ is less than $(1+\varepsilon) p\left|\bigcup_{i} S_{i}\right|$ we will have to apply union bound. From Claim 4.2, Claim 4.3 and following the argument given above, we have that at any stage of the algorithm the size of $\mathcal{X}$ is less than $(1+\varepsilon) p\left|\cup_{i} S_{i}\right|$ with probability at least $\left(1-\frac{\delta}{2 M}\right)$. So using the union bound we have our result.

Proof of Theorem 4.1. From Lemma 4.4 we know that the output of the algorithm FPS-Estimator is an $(\varepsilon, \delta)$-approximation of $\left|\bigcup_{i=1}^{M} S_{i}\right|$. All that is left to be shown is the upper bound on the worst case space and update time complexity.

From Lemma 4.4 we know that with probability $(1-\delta / 2)$ the size of $\mathcal{X}$ is less than $(1+\varepsilon) p\left|\bigcup_{i} S_{i}\right|$. Since we need $O(\log |\Omega|)$ to store an element of $\Omega$ the space complexity of FPS-Estimator is upper bounded by $(1+\varepsilon) p \cdot\left(\left|\bigcup_{i} S_{i}\right|\right) \cdot(\log |\Omega|)$ which is as claimed in the theorem.

Now let us consider the update time. Once again from Lemma 4.4 we know that with probability $(1-\delta / 2)$ the size of $\mathcal{X}$ is never more than $(1+\varepsilon) p\left|\bigcup_{i} S_{i}\right|$. Since we assume the sets in the stream are Delphic, so it takes only $O(\log |\Omega|)$ time to check the if condition in line 5 of Algorithm 1. Thus we see that the time spend in line 5- 8 is

$$
O\left(p \cdot\left(\bigcup_{i} S_{i} \mid\right) \cdot(\log |\Omega|)\right) .
$$

Also due to the fact that the sets are Delphic we can obtain a random sample in $O(\log |\Omega|)$ time. But to obtain $N_{i}$ distinct samples from $S_{i}$, from Theorem 3.4 we see that, probability that we have to draw more than $O\left(N_{i} \cdot \log N_{i} \cdot \log (2 M / \delta)\right)$ number of uniform samples from $S_{i}$ is at most $\delta / 2 M$. Thus, by union bound, with probability $(1-\delta / 2)$ for all $1 \leq i \leq M$ if we draw $O\left(N_{i} \cdot \log N_{i} \cdot \log (2 M / \delta)\right)$ number of uniform samples from $S_{i}$ we get $N_{i}$ distinct samples from $S_{i}$. Thus in the for loop, the line $7-8$ can be executed in $O\left(N_{i} \log N_{i} \cdot \log (M / \delta) \cdot \log |\Omega|\right)$ time with probability at least $(1-$ $\delta / 2)$. Since for all $i, N_{i}$ is less than the maximum size of $\mathcal{X}$ during the run of the algorithm so we have the bound on the update time complexity.

4.1.2 Algorithm APS-Estimator. We can now present the algorithm APS-Estimator and prove its correctness and analyse its complexity. This would prove Theorem 1.6.

Claim 4.5. For all $k$, after the (partial) stream $S_{1}, \cdots, S_{k}$ has been processed, by the algorithm APS-Estimator, for any $x \in \bigcup_{i=1}^{k} S_{i}$ the element $x$ is in $\mathcal{X}$ with probability $p$. 


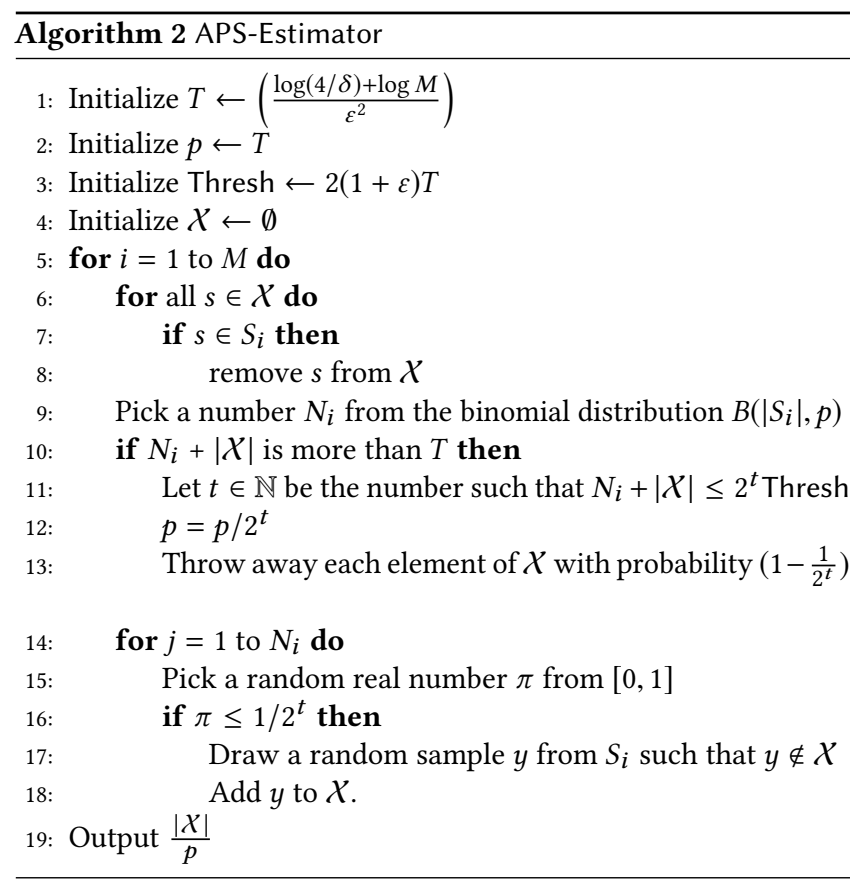

Proof. The proof is very similar to that of Claim 4.3. For the proof of Claim 4.5 we can perform induction on the number of items seen in the stream. Let us assume that after $j$ items $S_{1}, \cdots, S_{j}$ has been processed by the algorithm APS-Estimator, every item of $\bigcup_{i=1}^{j} S_{i}$ is in the set $\mathcal{X}$ with probability $p$. Now when the $S_{j+1}$ comes in the stream the algorithm (in for loop in line 6-8) will throw away all the items in $\mathcal{X}$ that are in $S_{j+1}$. So at that point (after line 8) all the elements in $\left(\bigcup_{i=1}^{j} S_{i}\right) \backslash S_{j+1}$ is in the set $\mathcal{X}$ with probability $p$.

In line 9 , a number $N_{j+1}$ is drawn from the binomial distribution $B\left(\left|S_{j+1}\right|, p\right)$. Note that drawing $N_{j+1}$ independent samples from $S_{j+1}$ is exactly same as selecting each element of $S_{j+1}$ independently with probability $p$. So if we had just added $N_{j+1}$ elements of $S_{j+1}$ to the set $\mathcal{X}$ we would be doing exactly same as in the case of Claim 4.3 and hence all elements of $\bigcup_{i=1}^{j+1} S_{i}$ would be in $\mathcal{X}$ independently with probability $p$.

The only thing that can be different is if the condition of the if loop (in line 10) is satisfied. In that case the $p$ is updated to $p / 2^{r}$ (in line 12) and since each element of the set $\mathcal{X}$ is independently thrown away with probability $\left(1-1 / 2^{r}\right)$, probability that an element is in $X$ is decreased by a factor of $2^{r}$. And after line 13, every element of $\left(\cup_{i=1}^{j} S_{i}\right) \backslash S_{j+1}$ is in the set $\mathcal{X}$ with probability $p$ (which is the new updated $p$ ). The for loop in line 14- 18 can be thought of as picking $N_{j+1}$ samples from $S_{j+1}$ and then throwing each of those samples with probability $\left(1-1 / 2^{r}\right)$. So each item of $S_{j+1}$ is also selected in $\mathcal{X}$ independently with the new updated probability $p$. So after processing the set $S_{j+1}$ we once again have the fact that every element of $\bigcup_{i=1}^{j+1} S_{i}$ in in $\mathcal{X}$ with probability $p$.

Proof of Theorem 1.6. Firstly, it is easy to see that the set $\mathcal{X}$ cannot ever cross Thresh, and so the space complexity is upper bounded by $O($ Thresh $\cdot(\log |\Omega|))$. The update time of the algorithm also is bounded by $O($ Thresh $\log ($ Thresh $) \cdot \log (2 M / \delta) \cdot(\log |\Omega|))$ (using the same argument as in Theorem 4.1). So the worst case space and update time complexity of APS-Estimator is as stated in the statement of Theorem 1.6. To complete the proof of Theorem 1.6 all we need to show is the correctness of the algorithm APS-Estimator.

Note that as the algorithm proceeds $p$ starts from $T$ and decreases to some value (say $T / 2^{k}$ ). Note that the final set of the samples in the set $\mathcal{X}$ is exactly what would have happened if we ran FPS-Estimator with $L=2^{k}$. Thus from Lemma 4.4 we have that if $\left|\bigcup_{i} S_{i}\right| \geq 2^{k}$ then with probability $\geq(1-\delta / 2)$ the algorithm APS-Estimator (as in algorithm FPS-Estimator) outputs an estimate that is between $(1-\varepsilon)\left|\cup_{i} S_{i}\right|$ and $(1+\varepsilon)\left|\cup_{i} S_{i}\right|$.

The output of the APS-Estimator is out of the desired bounds if $\left|\bigcup_{i=1}^{M} S_{i}\right|<2^{k}$, where the final value of $p$ is $T / 2^{k}$. Let us assume that $\left|\bigcup_{i=1}^{M} S_{i}\right|$ is $\geq 2^{r}$ and $<2^{r+1}$. So there must be a time when the $p$ value went from $\leq T / 2^{r}$ to $\geq T / 2^{r+1}$. Let that time frame be when the algorithm was processing item $S_{j}$ Now the reason algorithm updated $p$ at that time is because $\mathcal{X}+N_{j}$ must have gone bigger than Thresh.

By Lemma 4.4 if we ran the algorithm FPS-Estimator with the parameter $L$ set to $2^{r}$ then with probability $\geq(1-\delta / 2)$ the maximum size of $\mathcal{X}$ during the whole run of the algorithm is

$$
O\left((1+\varepsilon) \frac{\left|\bigcup_{i=1}^{M} S_{i}\right|}{2^{r} \varepsilon^{2}} \cdot(\log (4 / \delta)+\log M)\right) .
$$

And since we have assumed $\left|\bigcup_{i=1}^{M} S_{i}\right|$ is less than $2^{r+1}$, so with probability $\geq(1-\delta / 2)$ the maximum size of $\mathcal{X}$ during the whole run of the algorithm is less than $O\left(\frac{2(1+\varepsilon)}{\varepsilon^{2}} \cdot(\log (4 / \delta)+\log M)\right)$ which is Thresh.

So this event that is $\left|\bigcup_{i} S_{i}\right|<2^{k}$ while $p$ is updated to $T / 2^{k}$ can happen with probability at most $\delta / 2$. This proves that the algorithm APS-Estimator outputs an $(\varepsilon, \delta)$-approximation of $\left|\bigcup_{i=1}^{M} S_{i}\right|$.

\subsection{Klee's Measure Problem}

We return to Klee's measure problem in streaming setting and show that a straightforward application of Theorem 1.6 leads to the first space and update time efficient algorithm. As a first step, we show that the set Range(r) of a rectangle $\mathbf{r}$ is Delphic.

Lemma 4.6. For each rectangle $r$, Range $(r)$ belongs to Delphic family.

Proof. Note that Range $(\mathbf{r}) \subseteq[\Delta]^{d}$

(1) For a given $\mathbf{r}$, the size of the set is simply $\prod_{i=1}^{d}\left(b_{i}-a_{i}\right)$ can be computed in $O(d)$ time.

(2) To draw a uniform random sample $\left(x_{1}, \ldots x_{d}\right), x_{i}$ is sampled uniformly at random from $\left[a_{i}, b_{i}\right]$, which can be accomplished in $O(d \log \Delta)$

(3) Given $x=\left(x_{1}, \ldots x_{d}\right)$, we can check if $x \in \operatorname{Range}(\mathbf{r})$ by checking if for all $i, x_{i} \in\left[a_{i}, b_{i}\right]$, which can be accomplished in $O(d)$

Now, upon observing that each $r_{i}$ implicitly represents Range $\left(r_{i}\right)$, the following corollary immediately follows from Theorem 1.6. 
Corollary 1.8. There is a streaming algorithm that given any reals numbers $\varepsilon, \delta<1$, and a stream $\mathcal{R}=\left\langle\boldsymbol{r}_{1}, \boldsymbol{r}_{2}, \cdots \boldsymbol{r}_{M}\right\rangle$ where each $\boldsymbol{r}_{i}$ is a d-dimensional rectangle over $\Omega=\left[\Delta^{d}\right]$, computes an $(\varepsilon, \delta)$ approximation of $\operatorname{Volume}(\mathcal{R})$. The algorithm has worst case space $O\left(d(\log \Delta) \cdot \log (M / \delta) \cdot \varepsilon^{-2}\right)$ and update time complexity $O\left(d(\log \Delta) \cdot(\log (M / \delta))^{2} \log \log (M / \delta) \cdot \varepsilon^{-2} \log \varepsilon^{-1}\right)$.

The notion of range $\left[a_{i}, b_{i}\right]$ can be generalized to arithmetic progressions, which was studied previously by Pavan and Tirthpura for $d=1$. Our sampling model allows us to derive streaming algorithms for a more general model comprising of $d$-dimensional arithmetic progressions: Let $[a, b, c]$ represent the arithmetic progression with common difference $c$ in the range $[a, b]$, i.e., $a, a+c, a+2 c, a+j c$, where $j$ is the largest integer such that $a+j d \leq b$. Consider a stream $\mathcal{R}=\left\langle\mathbf{r}_{1}, \mathbf{r}_{2}, \ldots \mathbf{r}_{m}\right\rangle$ wherein each $\mathbf{r}_{i}=\left[a_{1}, b_{1}, c_{1}\right] \times \cdots \times\left[a_{d}, b_{d}, c_{d}\right]$ We generalize Range $(\mathbf{r})$ to denote the set of tuple $\left\{\left(x_{1}, \ldots x_{d}\right)\right\}$ where $a_{i} \leq x_{i} \leq b_{i}$ and $x_{i}=a_{i}+k \cdot c_{i}$ for some positive integer $\mathrm{k}$. Similarly, $\operatorname{Volume}(\mathcal{R})=\left|\bigcup_{i=1}^{m} \operatorname{Range}\left(\mathbf{r}_{i}\right)\right|$. To apply Theorem 1.6, we first show that Range(r) of a $d$-dimensional arithmetic progressions is Delphic.

Lemma 4.7. For each d-dimensional arithmetic progressions $\boldsymbol{r}$, the set Range( $\boldsymbol{r})$ belongs to Delphic family.

Proof. Note that Range $(\mathbf{r}) \subseteq[\Delta]^{d}$

(1) For a given $\mathbf{r}$, the size of the set is simply $\prod_{i=1}^{d}\left(\left\lfloor\frac{b_{i}-a_{i}}{c_{i}}\right\rfloor+1\right)$, which can be computed in $O(d)$ time.

(2) To draw a uniform sample $\left(x_{1}, \ldots x_{d}\right), x_{i}$ is sampled uniformly at random from $\left[a_{i}, b_{i}, c_{i}\right]$. Note that to sample from $\left[a_{i}, b_{i}, c_{i}\right]$, we first draw a number $k$ uniformly at random from $\left(\left\lfloor\frac{b_{i}-a_{i}}{c_{i}}\right\rfloor+1\right)$, and then return $a_{i}+k \cdot c_{i}$

(3) Given $x \stackrel{c_{i}}{=}\left(x_{1}, \ldots x_{d}\right)$, we can check if $x \in \operatorname{Range}(\mathbf{r})$ by checking if for all $i, x_{i} \in a_{i}+k \cdot c_{i}$ for some positive integer $\mathrm{k}$ and $x_{i} \leq b_{i}$, which can be accomplished in $O(d)$ time.

We can now invoke Theorem 1.6 to obtain the following result.

Corollary 1.10. There is a streaming algorithm that given any positive reals numbers $\varepsilon, \delta<1$, and a stream $\mathcal{R}=\left\langle\boldsymbol{r}_{1}, \boldsymbol{r}_{2}, \cdots \boldsymbol{r}_{M}\right\rangle$ consisting of d-dimensional arithmetic progressions, computes an $(\varepsilon, \delta)$ approximation of $\operatorname{Volume}(\mathcal{R})$. The algorithm has worst case space complexity $O\left(d(\log \Delta) \cdot \log (M / \delta) \cdot \varepsilon^{-2}\right)$ and worst case update time complexity $O\left(d(\log \Delta) \cdot(\log (M / \delta))^{2} \log \log (M / \delta) \cdot \varepsilon^{-2} \log \varepsilon^{-1}\right)$.

\subsection{Test Coverage Estimation}

We now focus on test coverage estimation problem and provide the proof for Corollary. We begin with the following lemma.

Lemma 4.8. For each $\boldsymbol{a}_{i}, \operatorname{Cov}\left(\boldsymbol{a}_{i}\right)$ belogns to Delphic family

Proof. Note that $\operatorname{Cov}\left(\mathbf{a}_{i}\right) \subseteq\{0,1\}^{t \log n+t}$.

(1) $\mid \operatorname{Cov}\left(\mathbf{a}_{i} \mid=\left(\begin{array}{l}n \\ t\end{array}\right)\right.$

(2) Drawing a uniform sample is simply drawing a $\log _{2}\left(\left(\begin{array}{c}n \\ t\end{array}\right)\right)$-bit strings uniformly at random, which can be accomplished in $O\left(\log \left(\left(\begin{array}{l}n \\ t\end{array}\right)\right)\right)$.

(3) Finally, to check if $x=(T, y) \in \operatorname{Cov}\left(\mathbf{a}_{i}\right)$, we simply compute the restriction of $a_{i}$ over $T$ and perform a string equivalence check.
Upon observing that each $\mathbf{a}_{i}$ implicitly represents $\operatorname{Cov}\left(\mathbf{a}_{i}\right)$, the following corollary immediately follows from Theorem 1.6

Corollary 1.11. There is a streaming algorithm APS-Estimator that given any reals numbers $\varepsilon, \delta<1$, and a stream $\mathcal{A}=\left\langle\boldsymbol{a}_{1}, \cdots, \boldsymbol{a}_{M}\right\rangle$, where $\boldsymbol{a}_{i} \in\{0,1\}^{n}$, computes an $(\varepsilon, \delta)$-approximation of $\left|\operatorname{Cov}_{t}(\mathcal{A})\right|$. The algorithm has space complexity $O\left(t(\log n) \cdot \log (M / \delta) \cdot \varepsilon^{-2}\right)$ and worst case update time complexity

$$
O\left(t(\log n) \cdot(\log (M / \delta))^{2} \log \log (M / \delta) \cdot \varepsilon^{-2} \log \varepsilon^{-1}\right) .
$$

A natural question one wonders is whether the space complexity of the above algorithm is tight. The following observation illustrates that the space complexity can be improved but at the cost of update time.

Observation 4.9. There is a streaming algorithm that given a stream $\mathcal{A}=\left\langle\boldsymbol{a}_{1}, \cdots, \boldsymbol{a}_{M}\right\rangle$, computes an $(\varepsilon, \delta)$-approximation of $\left|\operatorname{Cov}_{t}(\mathcal{A})\right|$. The algorithm takes $\left.O\left(t \log n+\varepsilon^{-2}\right) \log \frac{1}{\delta}\right)$ space and $O\left(n^{t}\right)$ update time.

Proof. For the item $\mathbf{a}_{i}$, produce a steam with $\left(\begin{array}{c}n \\ t\end{array}\right)$ elements $\mathbf{b} \in\{0,1\}^{t}$ that are covered by $\mathbf{a}_{i}$ and use the algorithm due to Kane, Nelson, and Woodruff [28] to compute the $F_{0}$ of the resulting stream. The reduction takes $O(t \log n)$ space and $O\left(n^{t}\right)$ time. The $\mathbb{F}_{0}$ computation on the resulting stream takes $\left.O\left(\left(t \log n+\varepsilon^{-2}\right) \log 1 / \delta\right)\right)$ and $O(1)$ processing time per item.

One wonders whether we can expliot the trade off between space and time complexity to arrive at an algorithm that has better space complexity than Corollary 1.11 and time complexity that is better than Observation 4.9. To this end, we present a hashing-based strategy that can replace the time complexity of $O\left(n^{t}\right)$ with linearly many calls to NP oracle.

Before, we state the result, we take a detour and formally introduce the notion of pairwise independent hash functions.

\section{Hashing-based Coverage Estimation}

We begin with an alternate view of $\operatorname{Cov}_{t}(a)$. We can view a as a string $a_{1} a_{2} \cdots a_{n} \in\{0,1\}^{n}$. Then,

$$
\begin{gathered}
\operatorname{Cov}_{t}(\mathbf{a})=\left\{\left\langle i_{1}, i_{2}, \cdots, i_{t} ; b_{1}, b_{2}, \cdots, b_{t}\right\rangle \mid i_{1}<i_{2}<\cdots<i_{t}\right. \\
\text { and } \left.a_{i_{j}}=b_{j} \forall 1 \leq j \leq t\right\} .
\end{gathered}
$$

Therefore, each element of $\operatorname{Cov}_{t}(\mathbf{a})$ can be seen as a binary string of length $(t+1) \log n$ and we will focus on the universe $\{0,1\}^{(t+1) \log n}$.

We will now prove the following theorem that improves upon the space complexity of Corollary 1.11 when allowed access to an NP oracle.

THEOREM 1.12. There is a streaming algorithm HashingEstimator with an NP set as an oracle that given a stream $\mathcal{A}=\left\langle\boldsymbol{a}_{1}, \cdots, \boldsymbol{a}_{M}\right\rangle$, and real numbers $0<\varepsilon, \delta<1$, where each $\boldsymbol{a}_{i} \in\{0,1\}^{n}$ computes an $(\varepsilon, \delta)$-approximation of $\left|\operatorname{Cov}_{t}(\mathcal{A})\right|$. The algorithm takes $O(t \log n$. $\left.\varepsilon^{-2} \cdot \log \frac{1}{\delta}\right)$ space and $\operatorname{poly}(n, t, 1 / \varepsilon, \log 1 / \delta)$ update time. Thus, if $\mathrm{P}=\mathrm{NP}$, algorithm will run in time $\operatorname{poly}(n, t, 1 / \varepsilon, \log 1 / \delta)$. 
Algorithm 3 can be viewed as an adaptation of Gibbons and Tirthapura's algorithm for $F_{0}$ estimation wherein every element of the stream $a$ represents the associated set $\operatorname{Cov}_{t}(a)$. The algorithm first selects a $T=O(\log (1 / \delta))$ pairwise independent hash functions. We then maintain two arrays of size $T$ : array of sketches $\mathcal{X}$ and an associated array of levels (represented as integers) $m$. In particular, corresponding to every hash function $H[i]$, we maintain an associated level $m[i]$ and the corresponding $\mathcal{X}[i]$. The core underlying idea is that at every point, $\mathcal{X}[i]$ consists of the all $y \in\{0,1\}^{(t+1) \log n}$ covered by the stream so far such that for each $y$, we have $H[i]_{m[i]}(y)=0^{m[i]}$. Furthermore, to avoid storing exponentially many elements, we ensure that $X[i]<$ thresh. To this end, we first check whether $\left|\left(H[i]_{m[i]}^{-1}\left(0^{m[i]}\right) \cap \operatorname{Cov}_{t}(a)\right) \cup \mathcal{X}[i]\right|$ is less than thresh, and in such a case, we increment the value of $m[i]$ until the check in line 9 succeeds. Note that whenever we increment the value of $m[i]$ in line 13, we also refine the set of $\mathcal{X}[i]$ to ensure that for all the elements $y \in \mathcal{X}[i]$, it is indeed the case that $H[i]_{m[i]}(y)=0^{m[i]}$.

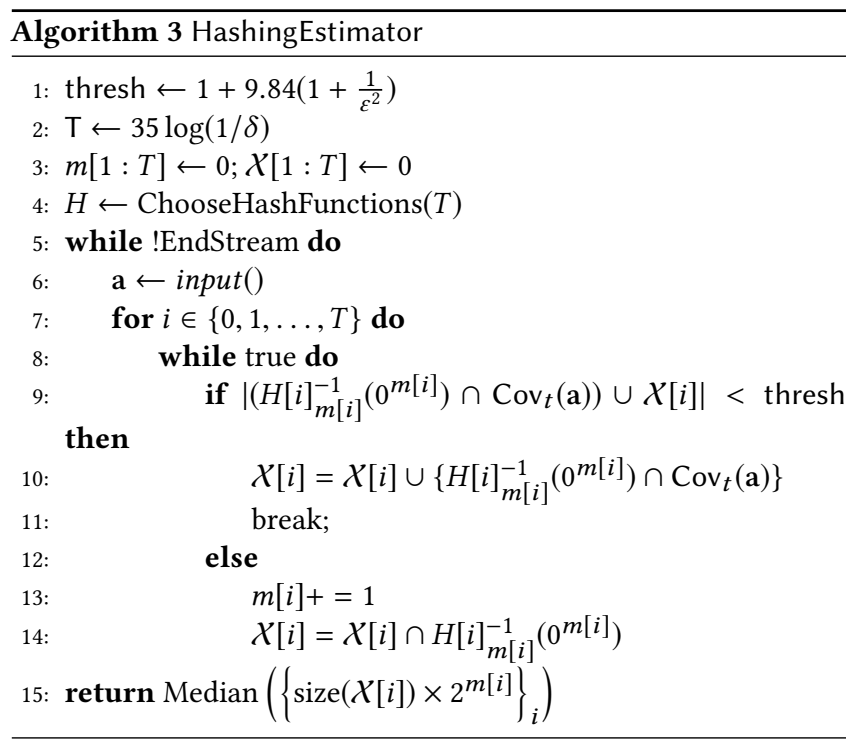

Lemma 4.10. Let $c=\operatorname{Median}\left(\left\{\operatorname{size}(X[i]) \times 2^{m[i]}\right\}_{i}\right)$. Then $\operatorname{Pr}\left[\frac{\operatorname{Cov}_{t}(\mathcal{A})}{1+\varepsilon} \leq c \leq \operatorname{Cov}_{t}(\mathcal{A})(1+\varepsilon)\right] \geq 1-\delta$

While allowing larger constants in the expression of thresh would allow us to use the arguments of Gibbons and Tirthapura [25], we provide an alternate proof building on Chakraborty, Meel, and Vardi [13] and Meel (r) Akshay [38] that allows us to obtain better constants. We believe the proof is of independent interest as it exploits the nested properties of the sets $H[i]_{m[i]}^{-1}\left(0^{m[i]}\right)$, which have shown to provide significant performance improvements in the context of model counting [13]. The proof is deferred to Appendix.

Now, the key question remains is of the time complexity. In essence, we are interested in the time complexity of the check in line 9. Observe that for all $y \in\{0,1\}^{(t+1) \log n}$, the check $y \in$ $\operatorname{Cov}_{t}(a)$ can be performed in $O((t+1) \log n)$ time. The following proposition follows from Lemma 3.7 of Bellare, Goldreich, and Petrank [4].

Lemma 4.11. Given a hash function $h \in \mathrm{H}_{\text {Teop }}(n, m)$ and $X$, there is a polynomial time algorithm $\mathcal{A}$ and $N P$ sets $M_{1}, M_{2}$ such that $\mathcal{A}^{M_{1}, M_{2}}(h, a, m, p, \mathcal{X})$ outputs 0 if $\left|\left(h^{-1}\left(0^{m}\right) \cap \operatorname{Cov}_{t}(a)\right) \cup \mathcal{X}\right| \geq p$ and $h^{-1}\left(0^{m}\right) \cap \operatorname{Cov}_{t}(a)$ otherwise. The algorithm makes $O(p(t+1) \log n)$ calls to NP oracle and uses $O(p(t+1) \log n)$ space.

Proof. The proof follows similar structure to Lemma 3.7 of [4]. $M_{1}=\left\{(a, h, m, p) \mid \exists y_{1}, \ldots y_{p}\right.$ such that $y_{1}, \ldots y_{p}$ are distinct and

$$
\left.\forall i \in[p]: y_{i} \in \operatorname{Cov}_{t}(a) \wedge h\left(y_{i}\right)=0^{m} \wedge y_{i} \notin \mathcal{X}\right\}
$$

$M_{2}=\left\{\left(a, h, m, p^{\prime}, i^{\prime}, j^{\prime}\right) \mid \exists y_{1} \prec y_{2} \ldots \prec y_{p^{\prime}}\right.$ such that

$y_{1} \cdot y_{p^{\prime}}$ are distinct and $\forall i \in\left[p^{\prime}\right]: y_{i} \in \operatorname{Cov}_{t}(a) \wedge$

$h\left(y_{i}=0^{m} \wedge\left(i^{\prime} \leq p^{\prime}\right) \wedge\left(j^{\prime} \leq\left|y_{i^{\prime}}\right|\right.\right.$ and $j^{\prime}$-th bit of $y_{i^{\prime}}$ is 0$\}$

Note that $\prec$ denotes a total ordering on $\{0,1\}^{(t+1) \log n}$. The algorithm $\mathcal{A}$ first invokes $M_{1}$ to output 0 if $\mid\left(h^{-1}\left(0^{m}\right) \cap \operatorname{Cov}_{t}(a) \cup \mathcal{X} \mid<p\right.$. Otherwise, $\mathcal{A}$ queries $M_{2}$ to determine all the $y \in\left(h^{-1}\left(0^{m}\right) \cap\right.$ $\left.\operatorname{Cov}_{t}(a)\right) \cup \mathcal{X}$ bit by bit.

Observe that Lemma 4.10 and 4.11 imply the desired theorem.

It is worth remarking that the usage of SAT oracle does not necessarily imply that the algorithm HashingEstimator would not be able handle practical instances. The past three decades have witnessed a sustained development of algorithmic techniques that allow modern SAT solvers to handle problems involving millions of variables [36]. Furthermore, the queries to SAT oracle in HashingEstimator can be expressed as conjunction of CNF and XOR constraints, also known as CNF-XOR formulas. Owing to the critical importance of CNFXOR formulas in the context of hashing-based techniques for model counting $[12,13,51]$, there has been a renewed focus on the design of efficient techniques for CNF-XOR formulas [49, 50]. We leave design of practical tools for future work.

\subsection{Model Counting of DNF}

Consider a DNF formula $\varphi=T_{1} \vee T_{2} \ldots \vee T_{M}$ wherein each $T_{i}$ is a term defined a set of $n$ Boolean variables.. We denote the set of all satisfying assignments of $\varphi$ by $\operatorname{Sol}(\varphi)$. Given $\varphi$, the problem of model counting seeks to compute $|\operatorname{Sol}(\varphi)|$. We focus on the problem of model counting for DNF formulas in streaming setting, i.e., where the terms $T_{i}$ arrive one by one over a stream. We first begin with the following lemma.

Lemma 4.12. For each $T_{i}$, Sol $\left(T_{i}\right)$ belongs to Delphic family.

Proof. Note that $\operatorname{Sol}\left(T_{i}\right) \in\{0,1\}^{n}$. Let $\left|T_{i}\right|$ denote the size of the term $T_{i}$

- $\operatorname{Sol}\left(T_{i}\right)=2^{n-\left|T_{i}\right|}$, which can computed in $O(n)$ time.

- Drawing a uniform sample from Sol $\left(T_{i}\right)$ is simply draw $n-$ $\left|T_{i}\right|$ bits uniformly at random, which can be accomplished in $O(n)$ time.

- Finally, check $x \in \operatorname{Sol}\left(T_{i}\right)$ can be accomplished in $O(n)$ time.

Since each $T_{i}$ implicitly represents $\operatorname{Sol}\left(T_{i}\right)$, the following corollary follows from Theorem 1.6. 
Corollary 1.13. There is a streaming algorithm that given any positive reals numbers $\varepsilon, \delta<1$, and a stream $\left\langle T_{1}, T_{2}, \ldots T_{M}\right\rangle$ of terms over $n$ variables, computes an $(\varepsilon, \delta)$-approximation of $\mid \operatorname{Sol}(\varphi \mid)$ wherein $\varphi=T_{1} \vee T_{2} \vee \ldots \vee T_{M}$. The algorithm takes $O\left(n \cdot \log (M / \delta) \cdot \varepsilon^{-2}\right)$ space and $O\left(n \cdot(\log (M / \delta))^{2} \log \log (M / \delta) \cdot \varepsilon^{-2} \log \varepsilon^{-1}\right)$ update time.

\section{CONCLUSION AND FUTURE OUTLOOK}

To summarize, our investigations led us to design a surprisingly simple yet efficient scheme for computation of size of union of sets belonging to Delphic family. We then show that the notion of Delphic sets can capture three fundamental problems in streaming setting: Klee's measure problem, coverage estimation problem, and DNF counting. For each of these problems, we provide efficient streaming algorithms.

Crucially, we believe the simplicity of our scheme should make it amenable to practical implementation and adoption. From technical perspective, we sketch out three directions of interest:

Generalization of Delphic Sets In this work, we limited our focus to three problems to showcase the generalizability of the notion of Delphic sets. As a future work, an interesting direction of work would be to study other streaming problems that reduce to Delphic sets. To this end, one line of work would be to relax the requirement of $O(\log |\Omega|)$ time to $O(\log |\Omega|)^{O(1)}$ for membership, counting, and sampling queries.

Higher Moments the computation of union of sets corresponds to $\mathbb{F}_{0}$ (0-th frequency moment) estimation. In this context, a natural question would be whether we can generalize our sampling-based strategy for $\mathbb{F}_{k}$, i.e., k-th frequency moment estimation.

Beyond Insertion-Only Streams The framework presented in this paper handles insertion only streams. The past two decades have witnessed a long line of work on richer turnstile models that allow deletion. Therefore, an interesting direction of future work would be to explore sampling-based framework for turnstile model.

Complexity independent of $\mathbf{M}$ The space and time complexity of APS-Estimator has logarithmic dependence on $M$, which is in line with the $O^{*}(1)$ notation introduced by Tirthapura and Woodruff [56]. However, observe that we can cast the distinct element problem as a special case of union of Delphic sets wherein every set is simply a singleton. In case of distinct element problem, the algorithms without dependence on $M$ are known. Therefore, an interesting direction for future work would be to investigate whether samplingbased framework can lead to algorithms whose space and update time complexity are independent of $M$.

Acknowledgments. We thank the anonymous reviewers of PODS 21 for valuable comments. Meel was supported in part by $\mathrm{Na}-$ tional Research Foundation Singapore under its NRF Fellowship Programme[NRF-NRFFAI1-2019-0004 ] and AI Singapore Programme [AISG-RP-2018-005], and NUS ODPRT Grant [R-252-000-685-13]. Vinodchandran was supported in part by NSF CCF-184908 and NSF HDR:TRIPODS-1934884 awards.

\section{REFERENCES}

[1] Dimitris Achlioptas and Panos Theodoropoulos. 2017. Probabilistic model counting with short XORs. In Proc. of SAT. Springer, 3-19.

[2] Noga Alon, Yossi Matias, and Mario Szegedy. 1999. The Space Complexity of Approximating the Frequency Moments. F. Comput. Syst. Sci. 58, 1 (1999), 137-147. https://doi.org/10.1006/jcss.1997.1545

[3] Ziv Bar-Yossef, Ravi Kumar, and D. Sivakumar. 2002. Reductions in streaming algorithms, with an application to counting triangles in graphs. In Proc. of SODA. ACM/SIAM, 623-632.

[4] M. Bellare, O. Goldreich, and E. Petrank. 2000. Uniform Generation of NPwitnesses using an NP-oracle. Information and Computation 163, 2 (2000), 510526.

[5] Jon Louis Bentley. 1977. Algorithms for Klee's rectangle problems. Technical Report. Technical Report, Computer.

[6] Valentin Tertius Bickel, Jordan Aaron, Andrea Manconi, Simon Loew, and Urs Mall. 2020. Impacts drive lunar rockfalls over billions of years. Nature Communications 11, 2862 (2020).

[7] Vladimir Braverman and Rafail Ostrovsky. 2010. Recursive Sketching For Frequency Moments. CoRR abs/1011.2571 (2010).

[8] Karl Bringmann and Tobias Friedrich. 2010. Approximating the volume of unions and intersections of high-dimensional geometric objects. Comput. Geom. 43, 6-7 (2010), 601-610.

[9] Renée C Bryce and Charles J Colbourn. 2009. A density-based greedy algorithm for higher strength covering arrays. Software Testing, Verification and Reliability 19, 1 (2009), 37-53.

[10] Mengchu Cai, Dinesh Keshwani, and Peter Z Revesz. 2000. Parametric rectangles: A model for querying and animation of spatiotemporal databases. In International Conference on Extending Database Technology. Springer, 430-444.

[11] J Lawrence Carter and Mark N Wegman. 1977. Universal classes of hash functions. In Proceedings of the ninth annual ACM symposium on Theory of computing. ACM, 106-112.

[12] S. Chakraborty, K. S. Meel, and M. Y. Vardi. 2013. A Scalable Approximate Model Counter. In Proc. of CP. 200-216.

[13] S. Chakraborty, K. S. Meel, and M. Y. Vardi. 2016. Algorithmic Improvements in Approximate Counting for Probabilistic Inference: From Linear to Logarithmic SAT Calls. In Proc. of IFCAI.

[14] Timothy M Chan. 2010. A (slightly) faster algorithm for Klee's measure problem. Computational Geometry 43, 3 (2010), 243-250.

[15] Moses Charikar, Kevin C. Chen, and Martin Farach-Colton. 2004. Finding frequent items in data streams. Theor. Comput. Sci. 312, 1 (2004), 3-15.

[16] Eric Y Chen and Timothy M Chan. 2005. Space-efficient algorithms for KleeâĂŹs measure problem. algorithms 3, 5 (2005), 6 .

[17] Bogdan S Chlebus. 1998. On the KleeâĂŹs measure problem in small dimensions. In International Conference on Current Trends in Theory and Practice of Computer Science. Springer, 304-311.

[18] David M. Cohen, Siddhartha R. Dalal, Michael L. Fredman, and Gardner C. Patton. 1997. The AETG system: An approach to testing based on combinatorial design. IEEE Transactions on Software Engineering 23, 7 (1997), 437-444.

[19] Jeffrey Considine, Feifei Li, George Kollios, and John Byers. 2004. Approximate aggregation techniques for sensor databases. In Proceedings. 20th International Conference on Data Engineering. IEEE, 449-460.

[20] Pedro J. Copado-MÃlndez, Carlos Pozo, Gonzalo Guillãln-GosÃąlbez, and Laureano Jimãlnez. 2016. Enhancing the $\varepsilon$-constraint method through the use of objective reduction and random sequences: Application to environmental problems. Computers \& Chemical Engineering 87 (2016), 36 - $48 . \quad$ https: //doi.org/10.1016/j.compchemeng.2015.12.016

[21] Graham Cormode and Shanmugavelayutham Muthukrishnan. 2003. Estimating dominance norms of multiple data streams. In European Symposium on Algorithms. Springer, 148-160.

[22] P. Dagum, R. Karp, M. Luby, and S. Ross. 2000. An optimal algorithm for Monte Carlo estimation. SIAM fournal on computing 29, 5 (2000), 1484-1496.

[23] Nilesh Dalvi and Dan Suciu. 2007. Efficient query evaluation on probabilistic databases. The VLDB fournal 16, 4 (2007), 523-544.

[24] Michael L Fredman and Bruce Weide. 1978. On the complexity of computing the measure of U[ai, bi]. Commun. ACM 21, 7 (1978), 540-544.

[25] Phillip B Gibbons and Srikanta Tirthapura. 2001. Estimating simple functions on the union of data streams. In Proceedings of the thirteenth annual ACM symposium on Parallel algorithms and architectures. 281-291.

[26] Joachim Gudmundsson and Rasmus Pagh. 2017. Range-Efficient Consistent Sampling and Locality-Sensitive Hashing for Polygons. In 28th International Symposium on Algorithms and Computation, ISAAC 2017, December 9-12, 2017, Phuket, Thailand (LIPIcs), Yoshio Okamoto and Takeshi Tokuyama (Eds.), Vol. 92. Schloss Dagstuhl - Leibniz-Zentrum für Informatik, 42:1-42:13.

[27] Piotr Indyk and David P. Woodruff. 2005. Optimal approximations of the frequency moments of data streams. In Proc. of STOC. ACM, 202-208.

[28] Daniel M. Kane, Jelani Nelson, and David P. Woodruff. 2010. An optimal algorithm for the distinct elements problem. In Proc. of PODS. ACM, 41-52. 
[29] David R Karger. 2001. A randomized fully polynomial time approximation scheme for the all-terminal network reliability problem. SIAM review 43, 3 (2001), 499522.

[30] R.M. Karp and M. Luby. 1983. Monte-Carlo algorithms for enumeration and reliability problems. Proc. of FOCS (1983).

[31] Richard M Karp, Michael Luby, and Neal Madras. 1989. Monte-Carlo approximation algorithms for enumeration problems. Fournal of Algorithms 10, 3 (1989), 429 - 448. https://doi.org/10.1016/0196-6774(89)90038-2

[32] Victor Klee. 1977. Can the Measure of be Computed in Less than O (n log n) Steps? The American Mathematical Monthly 84, 4 (1977), 284-285.

[33] D Richard Kuhn, Raghu N Kacker, and Yu Lei. 2013. Introduction to combinatorial testing. CRC press.

[34] Iosif Lazaridis and Sharad Mehrotra. 2001. Progressive approximate aggregate queries with a multi-resolution tree structure. Acm sigmod record 30, 2 (2001), 401-412.

[35] Robert Mandl. 1985. Orthogonal Latin squares: an application of experiment design to compiler testing. Commun. ACM 28, 10 (1985), 1054-1058.

[36] Joao Marques-Silva, Inês Lynce, and Sharad Malik. 2009. Conflict-driven clause learning SAT solvers. In Handbook of satisfiability. ios Press, 131-153.

[37] Flávio Medeiros, Christian Kästner, Márcio Ribeiro, Rohit Gheyi, and Sven Apel. 2016. A comparison of 10 sampling algorithms for configurable systems. In 2016 IEEE/ACM 38th International Conference on Software Engineering (ICSE). IEEE, 643-654.

[38] Kuldeep S. Meel (r) S. Akshay. 2020. Sparse Hashing for Scalable Approximate Model Counting: Theory and Practice. In Proc. of LICS

[39] Kuldeep S Meel, Aditya A Shrotri, and Moshe Y Vardi. 2017. On Hashing-Based Approaches to Approximate DNF-Counting. In In Proc. of FSTTCS.

[40] Kuldeep S. Meel, Aditya A. Shrotri, and Moshe Y. Vardi. 2018. Not All FPRASs are Equal: Demystifying FPRASs for DNF-Counting. Constraints An Int. f. (12 2018).

[41] Kuldeep S. Meel, Aditya A. Shrotri, and Moshe Y. Vardi. 2019. Not All FPRASs are Equal: Demystifying FPRASs for DNF-Counting (Extended Abstract). In Proc of IFCAI.

[42] Changhai Nie and Hareton Leung. 2011. A survey of combinatorial testing. ACM Computing Surveys (CSUR) 43, 2 (2011), 1-29.

[43] Mark H Overmars and Chee-Keng Yap. 1991. New upper bounds in KleeâĂŹs measure problem. SIAM 7. Comput. 20, 6 (1991), 1034-1045.

[44] Dimitris Papadias, Panos Kalnis, Jun Zhang, and Yufei Tao. 2001. Efficient OLAP operations in spatial data warehouses. In International Symposium on Spatial and Temporal Databases. Springer, 443-459.

[45] A. Pavan and Srikanta Tirthapura. 2007. Range-Efficient Counting of Distinct Elements in a Massive Data Stream. SIAM 7. Comput. 37, 2 (2007), 359-379.

[46] A. Pavan (r) N.V. Vinodchandran (r) Arnab Bhattacharyya (r) Kuldeep S. Meel. 2021. Model Counting meets $F_{0}$ Estimation. In Proc. of PODS.

[47] Tobias Pett, Thomas Thüm, Tobias Runge, Sebastian Krieter, Malte Lochau, and Ina Schaefer. 2019. Product sampling for product lines: the scalability challenge. In Proceedings of the 23rd International Systems and Software Product Line Conference, SPLC 2019, Volume A, Paris, France, September 9-13, 2019. ACM, 14:1-14:6. https: //doi.org/10.1145/3336294.3336322

[48] Gokarna Sharma, Costas Busch, Ramachandran Vaidyanathan, Suresh Rai, and Jerry L. Trahan. 2015. Efficient transformations for Klee's measure problem in the streaming model. Computational Geometry 48, 9 (2015), 688 - 702. https: //doi.org/10.1016/j.comgeo.2015.06.007

[49] Mate Soos, Stephan Gocht, and Kuldeep S. Meel. 2020. Tinted, Detached, and Lazy CNF-XOR solving and its Applications to Counting and Sampling. In Proceedings of International Conference on Computer-Aided Verification (CAV).

[50] Mate Soos and Kuldeep S Meel. 2019. BIRD: Engineering an Efficient CNF-XOR SAT Solver and its Applications to Approximate Model Counting. In Proceedings of AAAI Conference on Artificial Intelligence (AAAI)(1 2019).

[51] L. Stockmeyer. 1983. The complexity of approximate counting. In Proc. of STOC. $118-126$.

[52] He Sun and Chung Keung Poon. 2009. Two improved range-efficient algorithms for $\mathrm{F}_{0}$ estimation. Theor. Comput. Sci. 410, 11 (2009), 1073-1080.

[53] Yufei Tao and Dimitris Papadias. 2004. Range aggregate processing in spatial databases. IEEE Transactions on Knowledge and Data Engineering 16, 12 (2004), 1555-1570.

[54] Keizo Tatsumi. 1987. Test case design support system. In Proc. International Conference on Quality Control (ICQC'87). 615-620.

[55] Thomas Thüm, Sven Apel, Christian Kästner, Ina Schaefer, and Gunter Saake. 2014. A classification and survey of analysis strategies for software product lines. ACM Computing Surveys (CSUR) 47, 1 (2014), 1-45.

[56] Srikanta Tirthapura and David P. Woodruff. 2012. Rectangle-efficient aggregation in spatial data streams. In Proc. of PODS. ACM, 283-294.

[57] Jan Vahrenhold. 2007. An in-place algorithm for Klee's measure problem in two dimensions. Information processing letters 102, 4 (2007), 169-174.

[58] David Woodruff. 2020. personal communication.
[59] Donghui Zhang, Alexander Markowetz, Vassilis J Tsotras, Dimitrios Gunopulos, and Bernhard Seeger. 2008. On computing temporal aggregates with range predicates. ACM Transactions on Database Systems (TODS) 33, 2 (2008), 1-39.

\section{APPENDIX}

We provide a Proof of Lemma 4.10. We first restate the lemma below.

Lemma 4.10. Let $c=\operatorname{Median}\left(\left\{\operatorname{size}(X[i]) \times 2^{m[i]}\right\}_{i}\right)$. Then

$$
\operatorname{Pr}\left[\frac{\operatorname{Cov}_{t}(\mathcal{A})}{1+\varepsilon} \leq c \leq \operatorname{Cov}_{t}(\mathcal{A})(1+\varepsilon)\right] \geq 1-\delta
$$

Proof. While allowing larger constants in the expression of thresh would allow us to use the arguments of Gibbons and Tirthapura [25], we provide an alternate proof building on Chakraborty, Meel, and Vardi [13] and Meel@ Akshay [38] that allows us to obtain better constants. We believe the proof is of independent interest as it exploits the nested properties of the sets $H[i]_{m[i]}^{-1}\left(0^{m[i]}\right)$, which have shown to provide significant performance improvements in the context of model counting [1].

Let $Y=\left\{y_{1}, y_{2}, \ldots y_{F_{0}}\right\}$ be $F_{0}$ distinct elements covered by the input stream. For a fixed $i \in[|H|]$, let us use $\operatorname{Bad}_{i}$ to denote the event $\operatorname{size}(\mathcal{S}[i]) \times 2^{m[i]}$ does not lie in the interval

$$
\begin{gathered}
I_{\text {Good }}=\left[\frac{\left|\operatorname{Cov}_{t}(\mathcal{A})\right|}{1+\varepsilon},\left|\operatorname{Cov}_{t}(\mathcal{A})\right|(1+\varepsilon)\right] . \\
\text { Let Cnt }\langle i, j\rangle \\
\text { denote }\left|Y \cap H[i]_{j}^{-1}\left(0^{m[i]}\right)\right| \text {. For } j \in\{1, \ldots, n\} \text {, let } T_{i, j}
\end{gathered}
$$

denote the event that $\left(\mathrm{Cnt}_{\langle i, j\rangle} \leq\right.$ thresh -1$)$, and let $L_{i, j}$ and $U_{i, j}$

denote the events $\left(\operatorname{Cnt}_{\langle i, j\rangle}<\frac{\left|\operatorname{Cov}_{t}(\mathcal{A})\right|}{(1+\varepsilon) 2^{j}}\right)$ and $\left(\operatorname{Cnt}_{\langle i, j\rangle}>\frac{\left|\operatorname{Cov}_{t}(\mathcal{A})\right|}{2^{j}}\left(1+\frac{\varepsilon}{1+\varepsilon}\right)\right)$, respectively.

Now, for $\mathrm{Bad}_{i}$ to happen, either $L_{i, j}$ or $U_{i, j}$ must happen alongside the event $T_{i, j} \cap \overline{T_{i, j-1}}$. Thus, we obtain

$$
\operatorname{Pr}\left[\operatorname{Bad}_{i}\right] \leq \operatorname{Pr}\left[\bigcup_{j \in\{1, \ldots n\}}\left(\overline{T_{i, j-1}} \cap T_{i, j} \cap\left(L_{i, j} \cup U_{i, j}\right)\right)\right]
$$

Note that we only get an upper bound (and not an equality) above because the interval $I_{\text {Good }}$ considered has upper bound $\left|\operatorname{Cov}_{t}(\mathcal{A})\right|(1+$ $\varepsilon$ ), while $U_{i}$ and thresh are defined using the factor $\left(1+\frac{\varepsilon}{1+\varepsilon}\right) \leq 1+\varepsilon$.

Our next goal is to simplify this upper bound. Let $m^{*}$ be the smallest $j$ such that $\frac{\left|\operatorname{Cov}_{t}(\mathcal{A})\right|}{2^{j}}(1+\varepsilon) \leq$ thresh -1 . Now, by substituting the chosen value of thresh and simplifying, we obtain

$$
m^{*}=\left\lfloor\log _{2}\left|\operatorname{Cov}_{t}(\mathcal{A})\right|-\log _{2}\left(4.92 \cdot \rho \cdot\left(1+\frac{1}{\varepsilon}\right)^{2}\right)\right\rfloor
$$

We make use of the following simple but crucial observation:

$$
\forall j \in\{1, \ldots, n\}, T_{j} \Longrightarrow T_{j+1}
$$

Following [38], we can now state the claim that we can upper bound $\operatorname{Bad}_{i}$ by considering only five events, namely, $T_{i, m^{*}-3} L_{i, m^{*}-2}, L_{i, m^{*}-1}, L_{i, m^{*}}$ and $U_{i, m^{*}}$.

Claim 5.1. $\operatorname{Pr}\left[\operatorname{Bad}_{i}\right] \leq \operatorname{Pr}\left[T_{i, m^{*}-3}\right]+\operatorname{Pr}\left[L_{i, m^{*}-2}\right]+\operatorname{Pr}\left[L_{i, m^{*}-1}\right]+$ $\operatorname{Pr}\left[L_{i, m^{*}} \cup U_{i, m^{*}}\right]$

Proof. Let $\mu_{j}=\frac{\left|\operatorname{Cov}_{t}(\mathcal{A})\right|}{2^{j}}$. We make three observations, labeled $\mathrm{O} 1, \mathrm{O} 2$ and $\mathrm{O} 3$ below, which follow from the definitions of $\mathrm{m}^{*}$, 
thresh and $\mu_{j}$, and from the monotonicity of $\mathrm{Cnt}\langle i, j\rangle$ with respect to $j$ for a fixed $i$.

O1: $\forall j \leq m^{*}-3$, it is guaranteed that $\frac{\left|\operatorname{Cov}_{t}(\mathcal{A})\right|}{2^{j}(1+\varepsilon)} \geq$ thresh. From this it follows that (a) $T_{i, j} \cap U_{i, j}=\emptyset$ and (b) $T_{i, j} \cap L_{i, j}=T_{i, j}$ Therefore,

$$
\begin{aligned}
& \bigcup_{j \in\left\{1, \ldots m^{*}-3\right\}}\left(\overline{T_{i, j-1}} \cap T_{i, j} \cap\left(L_{i, j} \cup U_{i, j}\right)\right) \\
\subseteq & \bigcup_{j \in\left\{1, \ldots m^{*}-3\right\}}\left(\overline{T_{i, j-1}} \cap T_{j}\right) \\
\subseteq & \bigcup_{j \in\left\{1, \ldots m^{*}-3\right\}} T_{i, j} \subseteq T_{i, m^{*}-3}
\end{aligned}
$$

where the last containment follows from Equation 4 . Hence,

$\operatorname{Pr}\left[\bigcup_{j \in\left\{1, \ldots m^{*}-3\right\}}\left(\overline{T_{i, j-1}} \cap T_{i, j} \cap\left(L_{i, j} \cup U_{i, j}\right)\right)\right] \leq \operatorname{Pr}\left[T_{i, m^{*}-3}\right]$.

O2: For $j \in\left\{m^{*}-2, m^{*}-1\right\}$, it similarly follows that thresh $\leq$ $\frac{\left|\operatorname{Cov}_{t}(\mathcal{A})\right|}{2^{j}}\left(1+\frac{\varepsilon}{1+\varepsilon}\right)$, we have $T_{i, j} \cap U_{i, j}=\emptyset$. Since, $T_{i, j} \cap L_{i, j} \subseteq$ $L_{i, j}$, we have

$$
\begin{aligned}
& \operatorname{Pr}\left[\bigcup_{j \in\left\{m^{*}-2, m^{*}-1\right\}}\left(\overline{T_{i, j-1}} \cap T_{i, j} \cap\left(L_{i, j} \cup U_{i, j}\right)\right)\right] \\
\leq & \operatorname{Pr}\left[L_{i, m^{*}-2}\right]+\operatorname{Pr}\left[L_{i, m^{*}-1}\right] .
\end{aligned}
$$

O3: For $j \geq m^{*}$, it can be shown in the same vein that thresh $\geq$ $\frac{\left|\operatorname{Cov}_{t}(\mathcal{A})\right|}{2^{j}}\left(1+\frac{\varepsilon}{1+\varepsilon}\right)$, which implies that $\overline{T_{i, j}} \subseteq U_{i, j}$. Now, from
Equation 4, it follows that for all $j, \overline{T_{i, j}} \subseteq \overline{T_{i, j-1}}$. This implies that

$$
\begin{aligned}
& \operatorname{Pr}\left[\bigcup_{j \in\left\{m^{*}, \ldots|S|\right\}} \overline{T_{i, j-1}} \cap T_{i, j} \cap\left(L_{i, j} \cup U_{i, j}\right)\right] \\
& \quad \leq \operatorname{Pr}\left[\overline{T_{i, m^{*}}} \cup\left(\overline{T_{i, m^{*}-1}} \cap T_{i, m^{*}} \cap\left(L_{i, m^{*}} \cup U_{i, m^{*}}\right)\right)\right] \\
& \quad \leq \operatorname{Pr}\left[\overline{T_{i, m^{*}}} \cup L_{i, m^{*}} \cup U_{i, m^{*}}\right] \\
& \quad \leq \operatorname{Pr}\left[L_{i, m^{*}} \cup U_{i, m^{*}}\right]
\end{aligned}
$$

Claim 5.2. The following bounds hold:

(1) $\operatorname{Pr}\left[L_{i, m^{*}} \cup U_{i, m^{*}}\right] \leq \frac{1}{4.92}$

(2) $\operatorname{Pr}\left[L_{i, m^{*}-1}\right] \leq \frac{1}{10.84}$

(3) $\operatorname{Pr}\left[L_{i, m^{*}-2}\right] \leq \frac{1}{20.68}$

(4) $\operatorname{Pr}\left[T_{i, m^{*}-3}\right] \leq \frac{1}{62.5}$

Proof. Note that $\operatorname{Pr}\left[T_{i, j}\right]=\operatorname{Pr}\left[\mathrm{Cnt}_{\langle i, j\rangle} \leq\right.$ thresh $]$ and $\operatorname{Pr}\left[L_{i, j}\right]=$ $\operatorname{Pr}\left[\mathrm{Cnt}_{\langle i, j\rangle} \leq(1+\varepsilon)^{-1} \mu_{j}\right]$. Furthermore,

$\operatorname{Pr}\left[L_{i, j} \cup U_{i, j}\right]=\operatorname{Pr}\left[\mathrm{Cnt}_{\langle i, j\rangle}-\mu_{j} \mid \geq \frac{\varepsilon}{1+\varepsilon} \mu_{j}\right]$ To obtain bounds, we substitute values of $m^{*}$, thresh, $\mu_{j}$, and we apply Chebyshev and Payley-Zygmund inequalities.

Noting that $c=\operatorname{Median}\left(\left\{\operatorname{size}(\mathcal{S}[i]) \times 2^{m[i]}\right\}_{i}\right)$, we use the Chernoff bounds to obtain the desired bound. 